\title{
Prenatal therapy in Transient Abnormal Myelopoiesis: A Systematic Review
}

\author{
JA Tamblyn ${ }^{1}$, A Norton ${ }^{2}$, L Spurgeon ${ }^{1}$, V Donovan ${ }^{3}$, A Bedford Russell ${ }^{4}$, Bonnici J ${ }^{5}$, Perkins $K^{5}$, Vyas \\ $P^{5}$, I Roberts ${ }^{5}, M^{\prime}$ Kilby ${ }^{1}{ }^{3}$. \\ ${ }^{1}$ Centre for Women's \& Children's Health, College of Medical \& Dental Sciences, University of \\ Birmingham, B15 2TT, UK. \\ ²Birmingham Children's Foundation Trust, Steelhouse Lane, Birmingham, B4 6NH, UK. \\ ${ }^{3}$ Fetal Medicine Centre, Birmingham Women's Foundation Trust, Edgbaston, Birmingham, B15 2TG, \\ UK. \\ ${ }^{4}$ Department of Neonatal Paediatrics, Birmingham Women's Foundation Trust, Edgbaston, \\ Birmingham, B15 2TG, UK. \\ ${ }^{5}$ Department of Paediatrics, Children's Hospital and Molecular Haematology Unit, Weatherall \\ Institute of Molecular Medicine, Oxford University and John Radcliffe Hospital, Oxford, UK. \\ Corresponding Author: Professor Mark Kilby
}

Telephone: +44-121-627-2778

Email: m.d.kilby@bham.ac.uk 


\section{Objective}

To systematically review current evidence regarding prenatal diagnosis and management of transient abnormal myelopoiesis (TAM), in fetuses with Trisomy 21. A novel case of GATA1 positive TAM, in which following serial in-utero blood transfusion clinical improvement and postnatal remission were observed, is included.

\section{Search Strategy \& Data Collection}

A systematic search of electronic databases (inception to October 2014) and reference lists, hand searching of journals, and expert contact. All confirmed cases of prenatal TAM were included for analysis. Data on study characteristics, design, and quality were obtained.

\section{Results}

Of 73 potentially relevant citations identified, 22 studies were included, describing 39 fetuses. All studies included comprised single case or small cohort studies; overall quality was 'very low'. Fetal / neonatal outcome was poor; 12 stillbirths (30.8\%), 4 neonatal deaths (10.2\%) and 7 infant deaths (17.9\%). In two the pregnancy was terminated (5.1\%).

TAM was primarily detected in the third trimester $(79.4 \%)$, and in 14 a retrospective diagnosis was made postpartum. Ultrasound features indicative of TAM included hepatomegaly $+/$ - splenomegaly (79.5\%), hydrops fetalis (30.8\%), pericardial effusion (23.1\%), and aberrant liquor volume (15.4\%). When performed, liver function tests were abnormal in $91.6 \%$ cases.

\section{Conclusion}

Prenatal TAM presents a challenging diagnosis, and prognosis is poor, with consistently high mortality. A low threshold to measure haematological and biochemical markers is advised when clinical features typical of TAM are detected in the context of Trisomy 21. Larger prospective studies are warranted to accurately ascertain the role of GATA1 analysis, and potential value of prenatal therapy. 


\section{Introduction}

Trisomy 21 is the most common fetal autosomal Trisomy, affecting up to $1.3 \%$ of in-utero pregnancies(1) . These babies have excess mortality compared to euploid fetuses because of a number of co-morbidities.

Transient abnormal myelopoiesis (TAM) is a haematological disorder that exclusively affects babies with Trisomy 21 during fetal and neonatal life(2). Defined by the predominance of megakaryoblasts in peripheral blood, it presents in approximately $10 \%$ of new-born infants with Down Syndrome, and in most resolves within the first 3 months (up to $80 \%$ ) without treatment(3). However, TAM may progress to life-threatening illness in approximately $20 \%$ of cases; specifically secondary to liver dysfunction and/ or cardio-pulmonary disease(3). Furthermore, of the $20 \%$ of TAM cases that undergo neonatal recovery develop acute megakaryoblastic leukaemia (AML) in the first 4 years of life(4).

In TAM and AML, blast cells harbour an acquired mutation in exon 2 or 3 of the gene encoding GATA1, a key hematopoietic transcription factor associated with Down Syndrome(5-7). Following disease remission, GATA1 is undetectable(8). However, the current prospective definition of TAM specifies neither the percentage of blasts nor the role of GATA1 mutation analysis.

This review shall systematically assess all reported cases of prenatal-onset TAM with specific reference to its in-utero detection, investigation, and management. This group appear at particular risk of high mortality(9).

\section{Methods}

A case of prenatally diagnosed (GATA1 positive) TAM diagnosed in the second trimester is presented. We describe the in-utero management with serial blood transfusions with associated resolution of TAM. 
A systematic review of the literature high-lighting cases of prenatal-onset TAM was performed to assess the diagnostic features, investigations and prenatal management options.

Of 73 potentially citations, identified from the primary literature search using internationally recognised literature searching methodology (search terms; 'transient abnormal myelopoiesis', 'TAM', 'trisomy 21', 'down's syndrome', 'fetus*', 'prenatal', 'transient myeloproliferative disorder', 'an(a)emia*'), 22 papers were identified in the PubMed and Medline electronic databases describing 39 fetuses diagnosed with prenatal-onset TAM (Figure 1 and Table S1)(10-31). The predefined criteria were required for study inclusion:

(i) Diagnosis of fetal or neonatal Trisomy 21 / Down's Syndrome

(ii) Prenatal ultrasound features consistent with in utero onset of TAM

(iii) Haematological tests consistent with TAM (disease confirmation may be postnatal)

The assessment for inclusion, data extraction, and methodological quality evaluation were performed by two independent reviewers (JT, LS) prior to pooling and data analysis (JT).

\section{Case Report}

A 40 year old multiparous woman with a fetus with cytogenetically confirmed Trisomy 21, presented at 27 weeks gestation with a fetal ultrasound scan demonstrating an isolated large pericardial effusion, mild hepatomegaly with echogenic parenchymal foci, and significant abdominal ascites. There was little fetal movement observed on ultrasound, umbilical artery PI was elevated $(>95 \% \mathrm{Cl}$ for gestation) and there was absent flow in the ductus venosus during atrial contraction. A small ventriculospetal defect (VSD) was suspected. Fetal biometry demonstrated the baby was small for gestational age on customized growth charts $\left(<10^{\text {th }}\right.$ centile) but with normal maximal amniotic pool depth [MPD] $4.2 \mathrm{~cm}$. Percutaneous ultrasound-guided fetal blood sampling demonstrated haematological findings were consistent with a suspected diagnosis of TAM; leucocytosis (40.9 x10 $/ \mathrm{L}$; neutrophil count $0.4 \times 10^{9} / \mathrm{L}$, lymphocyte count $\left.0.8 \times 10^{9} / \mathrm{L}\right)$, thrombocytopenia $\left(31 \times 10^{9} / \mathrm{L}\right)$, 
fetal anaemia (haemoglobin $98 \mathrm{~g} / \mathrm{L}\left[<5^{\text {th }}\right.$ centile for gestation]; fetal direct coombs test negative) and a reticulocytosis (341 x 10\% $/ \mathrm{L}(13 \%)$. The fetal blood film (Figure 2 ) noted a circulating blast cell (38.4 x 10\% $/ \mathrm{L} ; 96 \%$ ) predominance. Fetal liver function tests were also abnormal (AST (59 iu/L) and low albumin $(27 \mathrm{~g} / \mathrm{L}))$. DNA analysis confirmed the presence of GATA1 mutation on sequencing. Figure 3 illustrates the 2 base pair frame-shift deletion of a TG in Exon 2 (HGVS nomenclature c.108_109delTG, p.Gly37Alafs*2) detected, which is considered a deleterious change with resultant loss of full length GATA1 protein.

Unique to this case was the decision to give supportive fetal therapy by serial in-utero transfusion of packed red cells and platelet concentrate as the fetus was mildly anaemic with thrombocytopenia (performed at diagnosis at 27 weeks $\left[\mathrm{Hb} \mathrm{98g/L} \mathrm{and} \mathrm{platelet} \mathrm{count} 31 \times 10^{9} / \mathrm{L}\right]$ ). This was repeated at 31 weeks gestation $\left(\mathrm{Hb} 111 \mathrm{~g} / \mathrm{L}\left[<5^{\text {th }}\right.\right.$ centile] and platelet count $\left.38 \times 10^{9} / \mathrm{L}\right)$, as the fetus had significantly reduced movements and appeared to have reaccumulated some abdominal ascites. The haematological indices subsequently improved with a significant reduction in the proportion of circulating blasts noted at repeat in-utero testing at 31 weeks. The improvement in ultrasound features (resolution of fetal effusions and reduction in hepatomatomegaly) suggests a combination of therapeutic and "spontaneous remission" prior to preterm delivery at 34 weeks. Delivery was expedited due to threatened pre-term birth with subsequent fetal distress on cardiotocograph monitoring. At birth, APGAR scores (9 at one and 5 minutes) and umbilical artery cord blood ( $\mathrm{pH}$ 7.26, base excess -0.7 ), were normal. A short 24-hour period of endotracheal ventilation was required with a further 56 hours of CPAP. The neonate required phototherapy until day 10 for mild hyperbilirubinemia (maximum total serum bilirubin $248 \mu \mathrm{mol} / \mathrm{l}$; conjugated $23 \mu \mathrm{mol} / \mathrm{l}$ ). Neonatal abdominal ultrasound confirmed the presence of mild hepatomegaly and splenomegaly, whilst cardiac echocardiography demonstrated a persistent small pericardial effusion, atrial septal and small ventricular septal defects. 
Further transfusions in the neonatal and infant period were not however indicated. The full blood count at birth showed a normal haemoglobin $(22.6 \mathrm{~g} / \mathrm{dl}), \mathrm{WBC}\left(12.8 \times 10^{9} / \mathrm{l}\right)$ and moderate thrombocytopenia (platelets $48 \times 10^{9} / \mathrm{I}$ ). Spontaneous resolution of all haematological parameters occurred over 14 days. The baby was discharged day 21 postnatal with nutrition support with multidisciplinary follow up, and remains well at present (at 9 months of age).

\section{Systematic Review}

Figure 1 summarises the process of literature identification and selection. Of the 73 potentially relevant citations identified from the primary literature search, 39 articles underwent detailed primary analysis, and 26 were excluded following identification as duplicates. A further 25 papers subsequently failed to meet the study inclusion criteria as detailed; 'incorrect study population and /or disorder' ( $n=19)$, 'review article' $(n=4)$, postnatal diagnosis $(n=3)$. In total 22 studies were included, describing 39 fetuses diagnosed with prenatal-onset TAM.

The study population includes 38 cases $(97.4 \%)$ with the diagnosis of Trisomy 21 confirmed with cytogenetic analysis; 31 (81.6\%) prenatal, and seven (18.4\%) postnatal. In outstanding case, IUFD occurred at 30 weeks and post-mortem analysis failed, however since Trisomy 21 was strongly suspected and both ultrasound and haematological markers consistent with TAM were evident (19).

Only 14 (39.5\%) were alive at most recent follow up (range; 2 weeks - 3 years), and there were 12 stillbirths (>24 weeks) (30.8\%), 4 neonatal deaths ( $\leq 28$ days) (10.2\%) and 7 infant deaths (17.9\%)(range; 31 days -3 years). In two cases the pregnancy was terminated (5.1\%); in one parental counselling included the possibility of TAM (21), whereas in the second severe liver dysfunction was identified. Post-mortem analysis enabled a retrospective diagnosis of TAM to be made(22). No cases of miscarriage ( $<24$ weeks) were reported. The gestation at delivery, (excluding cases of stillbirth ( $n=12)$, elective TOP $(n=2)$, and insufficient data $(n=2))$, was 8 cases delivering at term (>37 weeks) (34.8\%), 15 (65.2\%) were delivered preterm (<37 weeks), 9 (39.1\%) of which were at $<34$ weeks. 
'The ultrasound features evident in prenatal-onset TAM were hepatomegaly $+/$ - splenomegaly $(n=31 ; 79.5 \%)$, hydrops fetalis $(n=12 ; 30.8 \%)$, pericardial effusion $(n=9 ; 23.1 \%)$, aberrant liquor volume $(n=6 ; 15.4 \%)$, cardiac abnormalities $(n=5 ; 12.8 \%)$, fetal ascites $(n=4 ; 10.3 \%)$, pleural effusion $(n=3 ; 7.69 \%)$, and peripheral oedema ( $n=1 ; 2.56 \%)$. As illustrated in Figure 4 , in most cases $(n=28$; 71.8\%), at least 2 of these features were evident.'

In 13 cases (33.3\%) with hydrops fetalis and hepatomegaly, 12 (92.3\%) died either prenatally or in the early neonatal period. Of the remaining fatal cases of prenatal TAM $(n=11)$ (excluding termination of pregnancy [TOP]; $n=2)$, either hepatomegaly $(n=4 ; 36.3 \%)$ or hydrops fetalis $(n=8$; $54.5 \%)$ was evident in 10 cases (90.9\%). In the non-fatal cases of TAM ( $n=14)$, isolated hepatomegaly $(n=8 ; 57.1 \%)$ and hydrops fetalis $(n=1 ; 7.14 \%)$ were both detected. Prenatal isolated hepatomegaly is highly predictive of TAM. Hydrops fetalis is less common and but may be associated with a poor prognosis, when associated with liver dysfunction. Prenatal TAM presented most commonly in the third trimester $(n=31(79.4 \%))$. These data do not accurately reflect the timing of disease onset as detection was incidental in most cases following routine antenatal scan(19, 24, 26). Early, prenatal TAM detection is important as it permits parental counselling, and where appropriate, prenatal therapeutic support.

In only 26 cases (66.7\%) were prenatal FBS performed. Leucocytosis with blast cell predominance was confirmed in 25 (96.2\%) of these cases. In the remaining case, borderline leucocytosis and blaststosis was detected within the context of severe liver dysfunction. Post-mortem examination following $2^{\text {nd }}$ trimester TOP revealed excessive haematopoiesis and high megakaryocytes counts within the liver sinusoids, consistent with TAM(22). In 10 further cases leucocytosis and blast cell predominance were confirmed postnatally $(16,19,20,24,26,31)$. In 3 cases, leucocytosis without significant blast cell predominance $(10.9 \%$ and $9 \%)$ was observed, however in both there was a marked resolution of peripheral blast counts, which was concluded consistent with $\operatorname{TAM}(24,26)$. These findings support prenatal fetal blood sampling as a method of confirming suspected prenatal- 
onset of TAM in the context of Trisomy 21. No cases reported any direct procedure-related complications reported.

Thrombocytopenia screening was performed in 22 cases (84.6\%) prenatally, with 19 (86.4\%) confirmed thrombocytopenia. Liver function tests were performed in 12 cases (46.2 \%), 11 (91.6\%) of which were abnormal. There were 3 further cases which had abnormal liver function confirmed postnatally associated with prenatal hepatomegaly. Abnormal fetal liver function tests $(n=13)$ were associated with IUFD in 4 cases (30.8\%) and neonatal or infant death in 5 cases (38.5\%). In 1 case (7.69\%) TOP was performed. In 23 cases (59.0\%) liver function tests were not prospectively measured. Of particular note in the case reported here is the positive GATA1 mutation noted prenatally. This suggests that GATA1 status does not precede TAM. There are three cases for which GATA1 mutation analysis has been published within the context of prenatal-onset TAM(29). Of interest, is that Chen's case study did not identify a GATA1 mutation. In this case IUFD subsequently occurred at 36 weeks gestation, precluding repeat testing(27). In 24 cases (61.5\%) a prospective diagnosis of prenatal TAM was achieved. There were three cases of prenatal TAM in which intrauterine fetal blood transfusion was performed to treat fetal anaemia / thrombocytopenia. One fetus survived with spontaneous resolution of TAM, whilst the other two cases were fatal (one IUFD, one infant death (five months) $(29,30)$ ). In one case, therapeutic pericardiocentesis was performed for a fetus with a congenital cardiac anomaly and a large pericardial effusion. This fetus survived and entered spontaneous remission postnatally(14).

\section{Discussion}

The current incidence of TAM in infants with Down's Syndrome is $10 \%(3)$. This is likely to be an under-estimate(4). In keeping with previous reports, prenatal TAM is associated with a poor prognosis, particularly when associated with hydrops fetalis and liver dysfunction(25). It is not possible to identify positive prognostic features for prenatal TAM, particularly given the mean follow up time is too short to exclude progression to $\mathrm{AML}(6)$. Importantly, overall mortality rates are higher 
than those published recently for postnatal-onset TAM (up to 20\%)(32). It is important parents are counselled appropriately regarding the relatively poor prognosis of this condition.

Prenatal TAM had a consistent association with marked leucocytosis with blast cell predominance (96.2\%) and hepatic dysfunction (90.9\%). Thrombocytopenia was also evident in most cases prenatally $(81.8 \%)$. Fetal anaemia was only described in $2 / 7$ cases in the largest series $(28 \%)(25)$, which is consistent with previous reports of neonatal TAM(33).

In fetuses with Trisomy 21, a lower threshold in performing FBS, to investigate the haematological and liver function may facilitate earlier diagnosis of TAM (when abnormal ultrasound features are detected). Importantly, this systematic review does not demonstrate a definite association between hepatic impairment and fetal mortality, as reported previously(26). However, since biochemical testing was performed in less than $50 \%$ of cases, and the degree of dysfunction was not consistently reported, further studies are still warranted.

In a recent prospective analysis of 200 neonates with Trisomy 21, it was found that blood count and films noted $97.5 \%$ positive for circulating blasts. The findings strongly suggest additional more specific disease markers for TAM are required(33).

In this systematic review, GATA1 was negative in one case diagnosed with prenatal TAM(27). Furthermore, in neonates with Trisomy 21, GATA1 mutants have been detected when no other haematological abnormalities are detectable, denoting 'silent $\operatorname{TAM}^{\prime}(33)$. This phenomenon is not fully understood but suggests GATA1 status should be assessed only when there is a clinical suspicion of TAM, and that negative analysis does not fully preclude the potential diagnosis of TAM. There has been speculation as to whether a positive GATA1 status is predictive of AML predisposition. A retrospective review by Mansini et al, including 10 TAM [1-41 days age] and 4 AML [9-27 months age] patients reported $100 \%$ positive rates of GATA1 in all TAM cases. In the neonatal period, eight achieved complete spontaneous remission, with only one subsequently developing 
AML at 9 months ( 1 case lost to follow up)(6). These findings do not suggest positive GATA1 mutational analysis is predictive of TAM progression to AML. Furthermore, since all cases were GATA1 positive, an association with disease severity is also not evident. This is also reflected in the two cases reported here(29).

The subtype of GATA1 mutation has also not been found to be significant in predicting AML progression(34). Mansini et al did report that in one case of TAM which subsequently developed $A M L$, repeat mutation analysis revealed the presence of new mutations within the original mutation. Progression to AML may reflect the acquisition of additional mutations to GATA1. Whether disease protection was associated with negative GATA1 mutation analysis was unfortunately not measured (6). The persistence of positive GATA1 analysis postnatally in the case reported here is of uncertain relevance but of interest. Whether serial GATA1 analysis in previously positive patients following resolution of TAM is of clinical value is also important to understand.

Current evidence is limited as to the role of prenatal therapy in cases of TAM. In neonatal TAM, there are reports indicating that treatment with low-dose cytosine may be curative(3). In the context of severe hepatic dysfunction with or without cardiopulmonary embarrassment, early chemotherapeutic intervention with cytosine arabinoside is now recommended(35). No cases have been reported in which curative treatment has been commenced prenatally despite evidence of hepatic and cardio-pulmonary compromise. It is possible that supportive prenatal therapy may facilitate prolongation of gestation and enable postnatal therapy to be initiated. In the three cases where supportive fetal blood transfusion was given, fetal outcomes were however extremely disparate $(29,30)$. Furthermore, in-utero transfusion is not commonly warranted, and may be contraindicated if there is relative polycythaemia. Certainly the spontaneous regression of TAM observed in the case here, with improvement in both clinical and haematological parameters prenatally, is unusual and has not previously been reported. Since TAM may remit spontaneously, the intrauterine treatment given here may have contributed to the good outcome but we admit that 
this could be coincidental. However, in all other prenatally diagnosed cases with such a phenotype in-utero demise occurred $(29,30)$.

All cases included in this review were single case or small cohort studies ( $\leq 6$ cases). The overall quality of evidence is Cochrane GRADE classified 'very low'(36), which most likely reflects the low prevalence, and poor detection rates of fetal-onset TAM. Concerning bias, the overall risk was considered low, albeit selection and publication bias may both exist. For example, no reports of prenatal TAM which resulted in $2^{\text {nd }}$ trimester miscarriage were identified for this review.

There is currently insufficient evidence to make recommendations regarding prenatal supportive therapy in TAM. It is also not yet possible to define an exact role for positive prenatal GATA1 status, or the relevance of its persistence following disease remission. High quality, larger prospective observational studies (perhaps using an international registry) are required to more accurately define the diagnostic and prognostic markers of prenatal-onset TAM, and disease course. This would also help ascertain whether the timing of prenatal-TAM onset has important prognostic implications.

\section{Summary}

Prenatal TAM carries a poor prognosis, with high mortality rates consistently reported. Detection is reliant upon timely ultrasound detection and subsequent FBS. A low threshold for measuring haematological and biochemical markers is advised when clinical features typical of TAM are detected in a fetus with Trisomy 21 to facilitate detection and subsequent parental counselling. Higher quality evidence is required to accurately ascertain the roles of GATA1 analysis, and prenatal therapy. 


\section{Acknowledgements:}

The authors would like to acknowledge Steve Westwood from the Birmingham Children's

Foundation Trust flow cytometry laboratory who performed the immunophenotyping. Dr Jennifer

Tamblyn is funded by a Clinical Research Fellowship from Wellbeing of Women.

\section{Declaration of interest:}

The authors report no declarations of interest. 


\section{References}

1. Snijders RJ, Noble P, Sebire N, Souka A, Nicolaides KH. UK multicentre project on assessment of risk of trisomy 21 by maternal age and fetal nuchal-translucency thickness at 10-14 weeks of gestation. Fetal Medicine Foundation First Trimester Screening Group. Lancet. 1998;352(9125):3436.

2. Webb D, Roberts I, Vyas P. Haematology of Down syndrome. Archives of Disease in Childhood Fetal \& Neonatal Edition. 2007;92(6):F503-7.

3. Al-Kasim F, Doyle JJ, Massey GV, Weinstein HJ, Zipursky A. Incidence and treatment of potentially lethal diseases in transient leukemia of Down syndrome: Pediatric Oncology Group Study. Journal of pediatric hematology/oncology. 2002;24(1):9-13.

4. Zipursky A, Brown EJ, Christensen H, Doyle J. Transient myeloproliferative disorder (transient leukemia) and hematologic manifestations of Down syndrome. Clinics in Laboratory Medicine. 1999;19(1):157-67, vii.

5. Ahmed M, Sternberg A, Hall G, Thomas A, Smith O, O'Marcaigh A, et al. Natural history of GATA1 mutations in Down syndrome. Blood. 2004;103(7):2480-9.

6. Mansini AP, Rubio PL, Rossi JG, Gallego MS, Medina A, Zubizarreta PA, et al. Mutation characterization in the GATA-1 gene in patients with Down's Syndrome diagnosed with transient abnormal myelopoiesis or acute megakaryoblastic leukemia. Archivos argentinos de pediatria. 2013;111(6):532-6.

7. Mundschau G, Gurbuxani S, Gamis AS, Greene ME, Arceci RJ, Crispino JD. Mutagenesis of GATA1 is an initiating event in Down syndrome leukemogenesis. Blood. 2003;101(11):4298-300.

8. Wechsler J, Greene M, McDevitt MA, Anastasi J, Karp JE, Le Beau MM, et al. Acquired mutations in GATA1 in the megakaryoblastic leukemia of Down syndrome. Nature genetics. 2002;32(1):148-52.

9. Roy A, Cowan G, Vyas P, Roberts I. The impact of trisomy 21 on early human hematopoiesis. Cell Cycle. 2013;12(4):533-4.

10. Zerres K, Schwanitz G, Niesen M, Gembruch U, Hansmann M, Waldherr R. Prenatal diagnosis of acute non-lymphoblastic leukaemia in Down syndrome. Lancet. 1990;335(8681):117.

11. Foucar K, Friedman K, Llewellyn A, McConnell T, Aisenbrey G, Argubright K, et al. Prenatal diagnosis of transient myeloproliferative disorder via percutaneous umbilical blood sampling. Report of two cases in fetuses affected by Down's syndrome. American Journal of Clinical Pathology. 1992;97(4):584-90.

12. Hendricks SK, Sorensen TK, Baker ER. Trisomy 21, fetal hydrops, and anemia: prenatal diagnosis of transient myeloproliferative disorder? Obstetrics \& Gynecology. 1993;82(4 Pt 2 Suppl):703-5.

13. Macones GA, Johnson A, Tilley D, Wade R, Wapner R. Fetal hepatosplenomegaly associated with transient myeloproliferative disorder in trisomy 21. Fetal Diagnosis \& Therapy. 1995;10(2):1313.

14. Strobelt N, Ghidini A, Locatelli A, Vergani P, Mariani S, Biondi A. Intrauterine diagnosis and management of transient myeloproliferative disorder. American Journal of Perinatology. 1995;12(2):132-4.

15. Baschat AA, Wagner $T$, Malisius $R$, Gembruch U. Prenatal diagnosis of a transient myeloproliferative disorder in trisomy 21. Prenatal Diagnosis. 1998;18(7):731-6.

16. Hartung J, Chaoui R, Wauer R, Bollmann R. Fetal hepatosplenomegaly: an isolated sonographic sign of trisomy 21 in a case of myeloproliferative disorder. Ultrasound in Obstetrics \& Gynecology. 1998;11(6):453-5.

17. Siva S, Smoleniec J. Antenatal diagnosis of transient abnormal myelopoiesis associated with Down syndrome. Australian \& New Zealand Journal of Obstetrics \& Gynaecology. 1999;39(1):119-20.

18. Hirashima C, Eguchi Y, Kohmura Y, Minakami H, Sato I. Isolated pericardial effusion and transient abnormal myelopoiesis in a fetus with Down's syndrome. Journal of Obstetrics \& Gynaecology Research. 2000;26(4):303-6. 
19. Smrcek JM, Baschat AA, Germer U, Gloeckner-Hofmann K, Gembruch U. Fetal hydrops and hepatosplenomegaly in the second half of pregnancy: a sign of myeloproliferative disorder in fetuses with trisomy 21. Ultrasound in Obstetrics \& Gynecology. 2001;17(5):403-9.

20. Hamada H, Yamada N, Watanabe H, Okuno S, Fujiki Y, Kubo T. Hypoechoic hepatomegaly associated with transient abnormal myelopoiesis provides clues to trisomy 21 in the third-trimester fetus. Ultrasound in Obstetrics \& Gynecology. 2001;17(5):442-4.

21. Azancot A, Diehl R, Dorgeret S, Sebag G, Baumann C, Vuillard E, et al. Isolated pericardial effusion in the human fetus: a report of three cases. Prenat Diagn. 2003;23(3):193-7.

22. Vimercati A, Greco P, Gentile A, Ingravallo G, Loverro G, Selvaggi L. Fetal liver hyperechogenicity on sonography may be a serendipitous sign of a transient myeloproliferating disorder. Prenatal Diagnosis. 2003;23(1):44-7.

23. Robertson $M$, De Jong $G$, Mansvelt $E$. Prenatal diagnosis of congenital leukemia in a fetus at 25 weeks' gestation with Down syndrome: case report and review of the literature. Ultrasound in obstetrics \& gynecology : the official journal of the International Society of Ultrasound in Obstetrics and Gynecology. 2003;21(5):486-9.

24. Ogawa M, Hosoya N, Sato A, Tanaka T. Is the degree of fetal hepatosplenomegaly with transient abnormal myelopoiesis closely related to the postnatal severity of hematological abnormalities in Down syndrome? Ultrasound in Obstetrics \& Gynecology. 2004;24(1):83-5.

25. Hojo S, Tsukimori K, Kitade S, Nakanami N, Hikino S, Hara T, et al. Prenatal sonographic findings and hematological abnormalities in fetuses with transient abnormal myelopoiesis with Down syndrome. Prenatal Diagnosis. 2007;27(6):507-11.

26. Kikuchi A, Tamura N, Ishii K, Takakuwa K, Matsunaga M, Sudo N, et al. Four cases of fetal hypoechoic hepatomegaly associated with Trisomy 21 and transient abnormal myelopoiesis. Prenatal Diagnosis. 2007;27(7):665-9.

27. Chen CP, Tsai FJ, Chern SR, Chang TY, Hsu CY, Lin HH, et al. Prenatal diagnosis of $46, X X, \operatorname{DER}(13 ; 21)(Q 10 ; Q 10),+21$ and transient abnormal myelopoiesis in a fetus with hepatosplenomegaly and spontaneous resolution of fetal ascites. Taiwanese Journal of Obstetrics \& Gynecology. 2009;48(1):84-7.

28. Kim GJ, Lee ES. Prenatal diagnosis of transient abnormal myelopoiesis in a Down syndrome fetus. Korean Journal of Radiology. 2009;10(2):190-3.

29. Malin GL, Kilby MD, Velangi M. Transient abnormal myelopoiesis associated with Down syndrome presenting as severe hydrops fetalis: a case report. Fetal diagnosis and therapy. 2010;27(3):171-3.

30. Sukur YE, Gozukucuk M, Bayramov V, Koc A. Fetal hydrops and anemia as signs of Down syndrome. Journal of the Formosan Medical Association. 2011;110(11):716-8.

31. Mancuso A, Rijhsinghani A. Elevated delta OD 450 due to transient abnormal myelopoiesis in a Down syndrome fetus with hepatosplenomegaly on ultrasound. Prenat Diagn. 2014;34(3):299-301.

32. Cantor A. Myeloid proliferations associated with Down syndrome. J Hematopathol. 2014:1-

8.

33. Roberts I, Alford K, Hall G, Juban G, Richmond H, Norton A, et al. GATA1-mutant clones are frequent and often unsuspected in babies with Down syndrome: identification of a population at risk of leukemia. Blood. 2013;122(24):3908-17.

34. Alford KA, Reinhardt K, Garnett C, Norton A, Bohmer K, von Neuhoff C, et al. Analysis of GATA1 mutations in Down syndrome transient myeloproliferative disorder and myeloid leukemia. Blood. 2011;118(8):2222-38.

35. Gamis AS, Alonzo TA, Gerbing RB, Hilden JM, Sorrell AD, Sharma M, et al. Natural history of transient myeloproliferative disorder clinically diagnosed in Down syndrome neonates: a report from the Children's Oncology Group Study A2971. Blood. 2011;118(26):6752-9; quiz 996.

36. Higgins JP HJ. Cochrane Handbook for Systematic Reviews of Interventions. S G, editor [updated March 2011]. 
"The Corresponding Author has the right to grant on behalf of all authors and does grant on behalf of all authors, an exclusive licence (or non-exclusive for government employees) on a worldwide basis to the BMJ and co-owners or contracting owning societies (where published by the BMJ on their behalf), and its Licensees to permit this article (if accepted) to be published in Archives of Disease in Childhood and any other BMJ products and to exploit all subsidiary rights, as set out in our licence."

\section{Figure Legends}

Figure 1. Systematic Literature Search for Prenatal diagnosis of TAM (up to October 2014).

Figure 2. Blood films: $1 \mathrm{~A}$ : Fetal blood sample at 27 weeks gestation at $\times 100$ magnification, showing numerous blasts and nucleated red blood cells.

1B: Neonatal blood film on day 2 of postnatal life at x50 magnification, showing nucleated red cells, giant platelets (arrow), target cells and blast cells.

Figure 3. Exon 2 GATA1 gene mutation analysis of fetal DNA sourced from blood cells at 27 weeks gestation. Sanger sequence traces show that a TG dinucleotide deletion (arrow) has occurred in a proportion of cells in the fetal sample (bottom trace), which is absent in the wildtype (top trace) resulting in loss of full length of GATA1 protein. The double sequencing trace is due to presence of residual normal cells in the sample.

Figure 4. A summary of the total number of ultrasound features indicative of prenatal-TAM reported. 